\title{
Risk of upper gastrointestinal bleeding in a cohort of new users of low-dose ASA for secondary prevention of cardiovascular outcomes
}

\author{
Lucía Cea Soriano and Luis A. García Rodríguez* \\ Centro Español de Investigación Farmacoepidemiológica, Madrid, Spain
}

Edited by:

Angel Lanas, University of Zaragoza

Spain

Reviewed by:

A. T. Chan, Massachusetts General Hospital and Harvard Medical School, USA

F. H. Ng, Ruttonjee Hospital, China

*Correspondence:

Luis A. García Rodríguez, Spanish

Centre for Pharmacoepidemiologic

Research, C/Almirante 28, $2^{\circ} 28004$

Madrid, Spain.

e-mail: lagarcia@ceife.es

\begin{abstract}
The Health Improvement Network UK primary care database was used to identify a cohort of 38077 individuals aged 50-84 years with a first prescription of low-dose acetylsalicylic acid (ASA; 75-300 mg/day) for secondary prevention of cardiovascular or cerebrovascular events during 2000-2007. From this cohort, 169 incident cases of upper gastrointestinal bleeding (UGIB) were identified. Controls with no UGIB $(n=2000)$ were frequency-matched to the cases by age, sex, and follow-up time. A nested case-control analysis was performed to determine risk factors associated with UGIB. The incidence of UGIB was 1.1 per 1000 person-years $(95 \% \mathrm{Cl}, 1.0-1.3)$. Low-dose ASA users with a history of peptic ulcer disease had an increased risk of UGIB compared with those without (Relative Risk [RR], 4.59; 95\% Cl, 2.87-7.33). Concomitant use of ASA and clopidogrel (RR, $1.61 ; 95 \% \mathrm{Cl}, 0.85-3.05)$ or non-steroidal anti-inflammatory drugs (NSAIDs; RR, 2.92; 95\% Cl, 1.77-4.82) conferred an increased risk of UGIB compared with ASA monotherapy. Discontinuation of ASA therapy (RR: $0.71,95 \% \mathrm{Cl}, 0.42-1.20$ ) and PPI co-treatment given since the start of ASA therapy $(\mathrm{RR}, 0.56 ; 95 \% \mathrm{Cl}, 0.33-0.96)$ were associated with a reduced risk of UGIB. In conclusion, in a cohort of individuals receiving low-dose ASA for secondary prevention of cardiovascular or cerebrovascular events, patients with a history of peptic ulcer disease, or who were receiving clopidogrel or NSAIDs had an increased risk of UGIB. The prescription of PPI therapy at the initiation of low-dose ASA reduced the risk of UGIB by almost half.
\end{abstract}

Keywords: acetylsalicylic acid, case-control, secondary prevention, upper gastrointestinal bleeding

\section{INTRODUCTION}

Evidence-based guidelines recommend long-term use of low-dose acetylsalicylic acid (ASA; $75-150 \mathrm{mg} /$ day) for all patients with ischemic cardiovascular disease, unless contraindicated (Sacco et al., 2006; Smith et al., 2006; King et al., 2008). Low-dose ASA is known to be effective for the prevention of cardiovascular events in high-risk patients, reducing the risk of serious vascular events by around 25\% (Antithrombotic Trialists' Collaboration, 2002). However, low-dose ASA is also known to increase the risk of upper gastrointestinal bleeding (UGIB) (Lanas et al., 2007). Numerous observational studies have shown that the risk of upper gastrointestinal complications in patients receiving low-dose ASA is around twofold that in the general population (Weil et al., 1995; de Abajo and García Rodríguez, 2001; García Rodríguez et al., 2001).

Therefore, the cardiovascular benefits of low-dose ASA therapy should be weighed against the risk of any major bleeding, including UGIB. Risk factors for UGIB in patients taking ASA are not well characterized, but appear to include advanced age, history of peptic ulcer disease and concomitant use of NSAIDs (Patrono et al., 2005). A dose of ASA not greater than $81 \mathrm{mg}$ per day together with a gastroprotective medication is recommended for patients at high risk of bleeding (Bhatt et al., 2008).

The aims of this study were to estimate the incidence of UGIB in a UK primary care setting and, through a nested casecontrol analysis, establish the principal risk factors associated with an increased risk of UGIB among a cohort of individuals starting low-dose ASA therapy for secondary prevention of cardiovascular events.

\section{MATERIALS AND METHODS DATA SOURCE}

The Health Improvement Network (THIN) covers about $5 \%$ of the UK population, and is age, sex, and geographically representative (Bourke et al., 2004). Anonymized data on over 3 million patients are systematically recorded by participating primary care practitioners (PCPs) as part of their routine patient care and sent to THIN for use in research projects. The computerized information includes demographics, details from PCP visits, diagnoses, referrals to specialists and hospital admissions, results of laboratory tests, and a free-text section. Participating practices are required to record the indication for new courses of therapy, and prescriptions issued by the PCP are also recorded. The Read classification is used to code specific diagnoses (O'Neil et al., 1995; Stuart-Buttle et al., 1996), and a drug dictionary based on data from the MULTILEX classification is used to record prescriptions (First Data Bank, 2010). THIN has been extensively validated for use in pharmacoepidemiology (Lewis et al., 2007).

\section{STUDY DESIGN}

From THIN, a cohort of new users of low-dose ASA (75-300 mg/ day) for secondary prevention of cardiovascular ischemic diseases or cerebrovascular ischemic events who were aged 50-84 years 
between 1 January 2000 and 31 December 2007 was sampled. They were required to be registered for at least 2 years with their PCP and to have at least 1 year of computerized prescription history. We excluded all individuals with any of the following conditions: alcohol abuse, cancer, coagulopathies, esophageal varices, liver disease or Mallory-Weiss disease. The date of the first ever recorded prescription of low-dose ASA for secondary prevention of cardiovascular or cerebrovascular events was defined as the start date. All study cohort members $(N=38077)$ were followed for a mean of 4.0 years from the first day after the start date until the earliest of the following endpoints: UGIB event; diagnosis of alcohol abuse/ alcohol-related disease, cancer, coagulopathy, esophageal varices, liver disease, or Mallory-Weiss disease; reaching the age of 85 years; death or the end of the study period (30 October 2008). The study was approved by a Multicentre Research Ethics Committee.

\section{UGIB CASE ASCERTAINMENT}

After removing all personal identifiers, we reviewed the free-text comments and patient profiles which included demographic data and all clinical information for all individuals with a computerrecorded entry suggesting a UGIB event $(n=548)$. At this stage, patients were excluded if they had bleeding in a location other than the stomach or duodenum, or if they had no specific bleeding site recorded (unless it was recorded as "peptic ulcer"). Individuals were also excluded if they had gastrointestinal perforation, if the UGIB event was not confirmed upon further investigation or if they were not referred, or if they had a diagnosis of alcohol abuse/ alcohol-related disease, cancer, coagulopathy, esophageal varices, liver disease, or Mallory-Weiss disease. During the manual review, we also established the index date as the day of the first sign (e.g. bleeding) that led to the diagnosed outcome.

Following this review process, 169 patients were considered to be incident cases of UGIB (Figure 1). The site of bleeding was located in the stomach in $71(42 \%)$ patients, in the duodenum for $50(29.6 \%)$, in both sites for 27 (16.0\%), and in $21(12.4 \%)$ patients the specific site was only recorded as "peptic ulcer".

\section{SELECTION OF CONTROLS}

A date within the study period was generated at random for each member of the study cohort of 38077 individuals. If the random date was included in the individual's eligible person-time (follow-up period), we marked that person as an eligible control. The random date for controls was used as the index date in the nested case-control analysis. In total, 2000 controls, frequencymatched by sex, age, and follow-up time (interval between start date and index date), were sampled. The same exclusion criteria were applied to controls as to cases.

\section{COMORBIDITIES AND RISK FACTORS}

Information on comorbidities was collected from THIN from any time before the start date. Peptic ulcer antecedents was categorized into three groups: dyspepsia which included all diagnoses related with upper gastrointestinal symptoms; uncomplicated peptic ulcer; and complicated peptic ulcer defined as all individuals with bleeding or another complication. Data on potential risk factors, such as smoking, alcohol use (units per week), body mass index (BMI; kg/ $\mathrm{m}^{2}$ ) were collected from any time before the index date. In addition, data on the number of PCP visits, referrals and hospitalizations were collected for the year prior to the index date. Finally, information on drug exposure between the start date and the index date was reviewed.

Exposure to antiplatelet drugs (ASA, clopidogrel and dipyridamole), warfarin, nitrates, proton pump inhibitors (PPIs), histamine-2 receptor antagonists ( $\left.\mathrm{H}_{2} \mathrm{RAs}\right)$, oral steroids was classified as follows: current use, when the supply of the most recent prescription lasted until the index date or ended in the 30 days before the index date; past use, when supply of the most recent prescription ended 31-365 days before the index date; and nonuse when supply of the most recent prescription ended more than 365 days before the index date or there was no recorded use at any time between the start and index dates if that interval was smaller than 365 days. Additionally we evaluated the interaction between dual antiplatelet use (ASA + clopidogrel) and between ASA + NSAIDs, independently. We created five levels of exposure for each of these two variables as follows: Non-use of either agent (within the year prior to index date); use of both agents defined as current users of the two agents; current use of only one agent and non-use of the other agent within the year prior and vice versa; and remaining (combinations of recency of both agents).

To estimate the effect of low-dose ASA discontinuation on the risk of UGIB, we categorized ASA exposure using the following time windows: current use, when the supply of the most recent prescription lasted until the index date (assuming complete adherence) or ended in the 14 days before the index date; recent discontinuation when it ended 15-180 days before the index date; past discontinuation when it ended 181-365 days before the index date; and non-use when it ended more than 365 days before the index date. We also examined the reasons for lowdose ASA discontinuation and classified them into four mutually exclusive groups: lack of efficacy, defined as physician-initiated switching from low-dose ASA to another antiplatelet drug or to an anticoagulant such as warfarin with no evidence to suggest an adverse event; safety concerns, defined as evidence of adverse events related to low-dose ASA therapy (such as upper gastrointestinal disorders or UGIB), evidence of intolerance to low-dose ASA (allergy/urticaria), initiation of acid-suppressing drug treatment, or planned surgery; use of over-the-counter ASA, defined as when the PCP recorded that patients were receiving low-dose ASA in the absence of a computerized prescription or when the PCP noted that the patient was taking over-the-counter low-dose ASA; and non-adherence, defined as discontinuation in the absence of any of the above factors.

We subdivided current users of PPIs and $\mathrm{H}_{2} \mathrm{RAs}$ into two mutually exclusive groups: those who received their first prescription before or at the start date (defined as users initiating therapy at start date) and those who received their first prescription after the start date (defined as users initiating therapy after start date).

Exposure to NSAIDs was classified as follows: current use, when the supply of the most recent prescription lasted until the index date or ended in the 7 days before the index date (this shorter time window for NSAID current use was used to make it comparable with prior NSAID studies); past use, when it ended 8-365 days before the index date; and non-use when use ended 


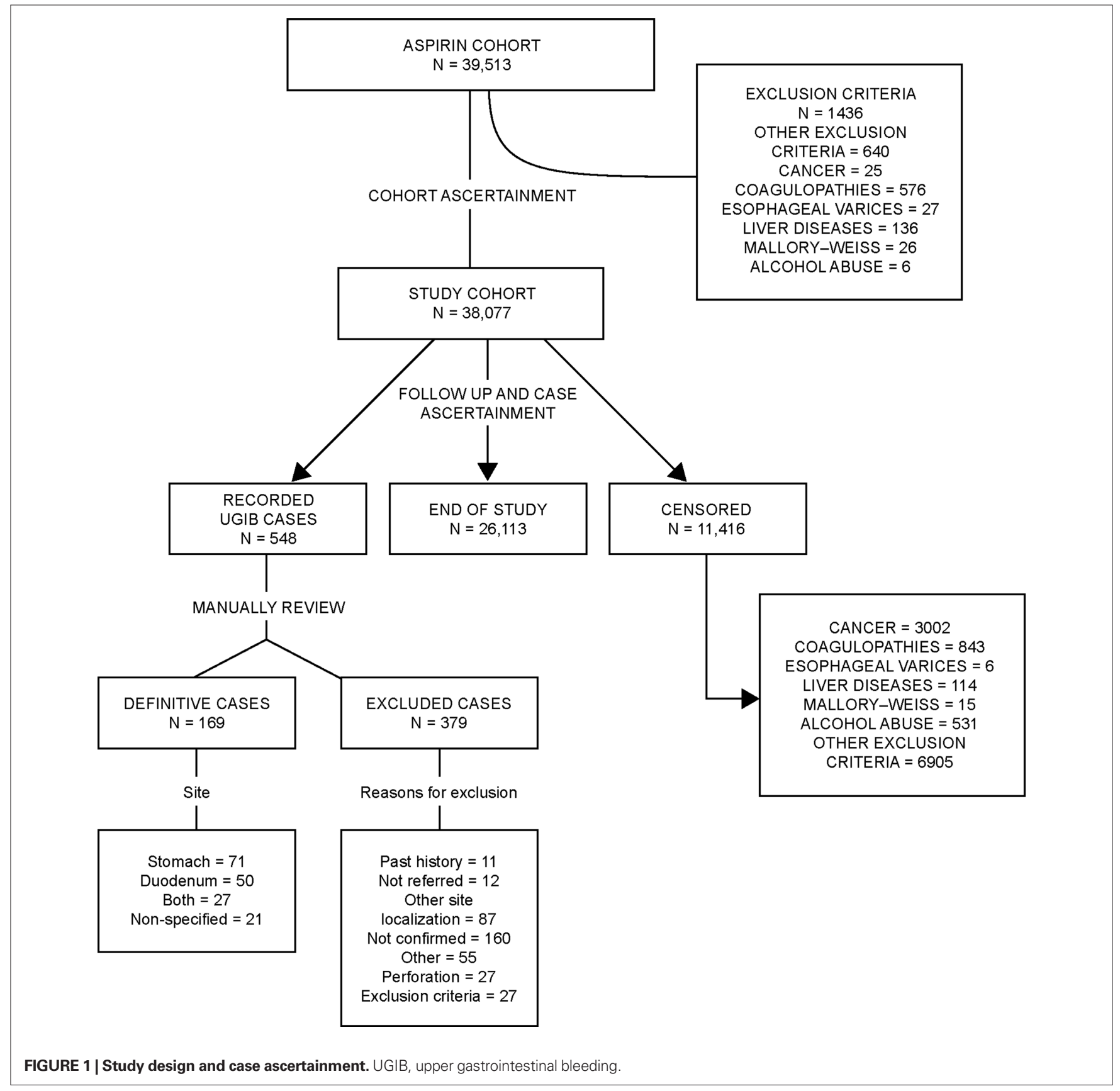

more than 365 days before the index date or there was no recorded use at any time between the start and index dates if that interval was smaller than 365 days. Current use of NSAIDs was further subdivided into the following categories: single, when there was use of only one NSAID in the 90 days before the index date, and multiple, when the patient used more than one NSAID in the 90 days before the index date. Dose- and duration-response were assessed in current users of single NSAIDs. Duration of treatment was computed by summing the number of days corresponding to consecutive prescriptions (allowing for an interval of 60 days or less between the end of one prescription and the start of the next one).

\section{STATISTICAL ANALYSIS}

The overall incidence of UGIB was estimated along with age- and sex-specific incidence estimates, and 95\% confidence intervals (CIs) were calculated. We also calculated the incidence of UGIB in subgroups of ASA users with or without a history of peptic ulcer disease before the start date. Kaplan-Meier survival analysis and log rank test was performed in the whole cohort as well as stratified by sex, age, and history of peptic ulcer disease.

Nested case-control analysis was performed to estimate the association between various risk factors and the occurrence of UGIB. Odds ratios (ORs) and 95\% CIs were calculated by unconditional multiple logistic regression models adjusted for age, sex, follow-up 
time, history of peptic ulcer disease, PCP visits, number of referrals and hospitalizations, and use of ASA, clopidogrel, warfarin, PPIs and NSAIDs (current, past, or no use). Under the study design of incidence density sampling, the OR is an unbiased estimator of the incidence rate ratio (RR). Statistical analyses were performed using Stata package version 11.0 (StataCorp LP, College Station, TX, USA).

\section{RESULTS \\ INCIDENCE OF UGIB}

We observed a crude incidence of UGIB of 1.12 per 1000 personyears (95\% CI, 0.96-1.30). The incidence of UGIB increased with age and was slightly higher among men 1.18 (95\% CI, 0.97-1.43) than women 1.05 (95\% CI, 0.83-1.33) (Figure 2). There was no significant association between UGIB and sex (log rank test $p$ value $=0.44)$, but older age was associated with UGIB $(>70$ years vs $\leq 70$ years; log rank test $p$ value $=0.0003$ ).

The incidence of UGIB was 3.53 per 1000 person-years $(95 \%$ CI, 2.53-4.92) among individuals with a previous diagnosis of peptic ulcer disease, 1.44 per 1000 person-years (95\% CI, 1.09-1.92) among individuals with a previous diagnosis of gastritis or dyspepsia, compared with 0.80 per 1000 person-years (95\% CI, 0.65-0.99) among individuals with none of these diagnoses.

The cumulative proportion of patients developing UGIB over time is presented in Figure 3. The incidence during the first year of follow-up was 1.71 (95\% CI, 1.34-2.20) per 1000 person-years and 0.93 (95\% CI, 0.77-1.13) per 1000 person-years during the remaining years of follow-up. Figures 4-6 present the cumulative proportion of patients developing UGIB according to sex, age, and history of peptic ulcer disease, respectively.

\section{RISK FACTORS FOR UGIB}

Associations between baseline characteristics and comorbidities and the risk of UGIB are shown in Table 1. Consumption of more than 25 units of alcohol per week was associated with a significant increase in the risk of UGIB (RR, 2.96; 95\% CI, 1.43-6.15; compared with non-use). The corresponding estimate of RR among current smokers was 1.33 (95\% CI, 0.80-2.21 compared with non-smokers. Prior diagnosis of peptic ulcer disease (with or without complication) was also associated with a significant increase in the risk of UGIB (RR, 4.59; 95\% CI, 2.87-7.33).

The RR of UGIB associated with current use of low-dose ASA was 1.63 (95\% CI, 0.85-3.13) (Table 2). The risk of UGIB was significantly increased in those receiving ASA 150-300 mg/day (RR, 2.65; 95\% CI, 1.17-5.97). The corresponding RR among those receiving $75 \mathrm{mg}$ /day was 1.54 (95\% CI, 0.80-2.97).

Current use of clopidogrel (with or without low-dose ASA) was associated with a significant increase in the risk of UGIB compared with non-use (RR, 1.90; 95\% CI, 1.12-3.22). Current users of dual antiplatelet therapy (ASA and clopidogrel) had a significantly higher risk of UGIB than individuals using neither antiplatelet therapy (RR, 3.31; 95\% CI, 1.18-9.24) and a non-significant increase in the risk of UGIB compared with those using low-dose ASA monotherapy (RR, 1.61; 95\% CI, 0.85-3.05).

Recent discontinuers of low-dose ASA had a lower risk of UGIB compared with those who continued low-dose ASA therapy (RR, 0.71; 95\% CI, 0.42-1.20; Table 3). When we examined the risk of

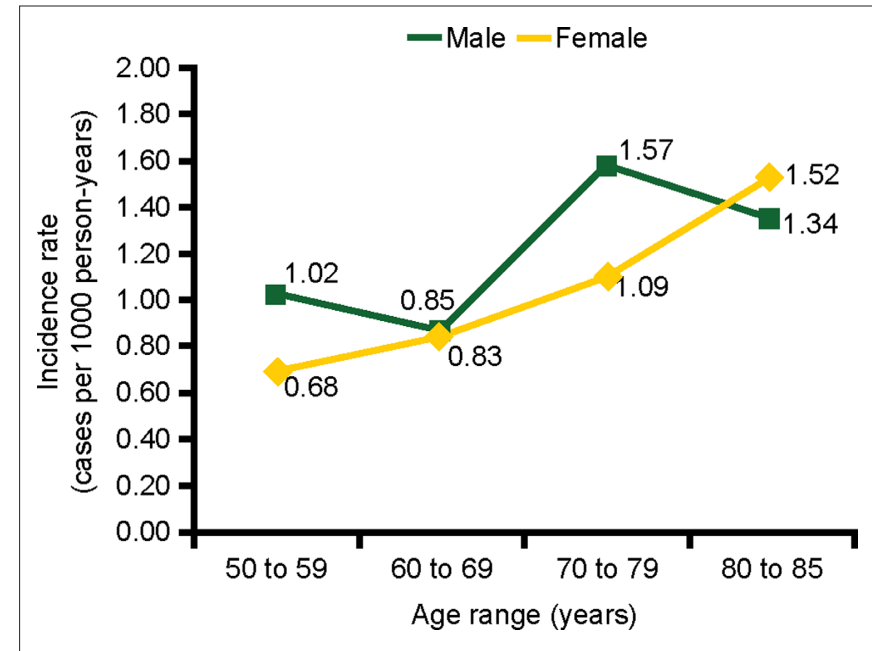

FIGURE 2 | Incidence of UGIB by age and sex in a cohort of low-dose ASA users. ASA, acetylsalicylic acid; UGIB, upper gastrointestinal bleeding.

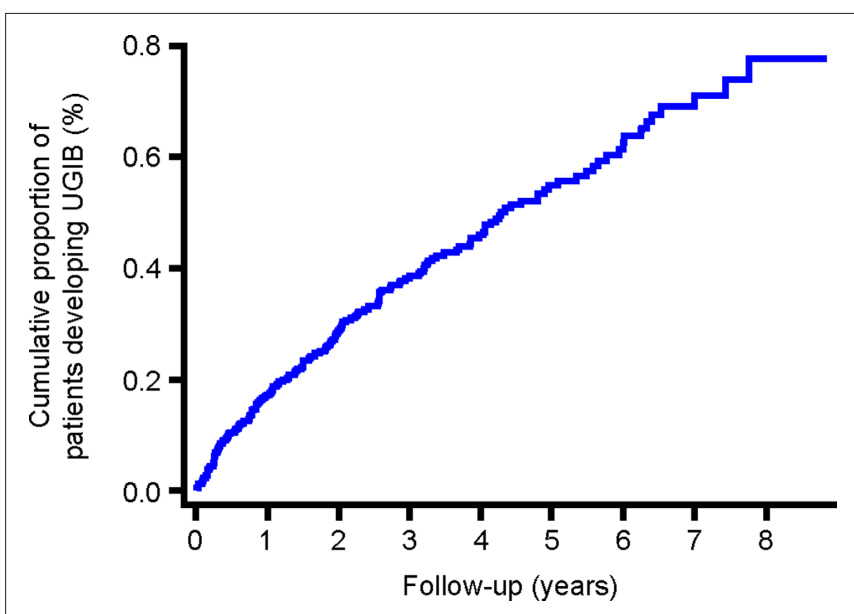

FIGURE 3 | Cumulative proportion of patients developing UGIB during follow-up. UGIB, upper gastrointestinal bleeding.

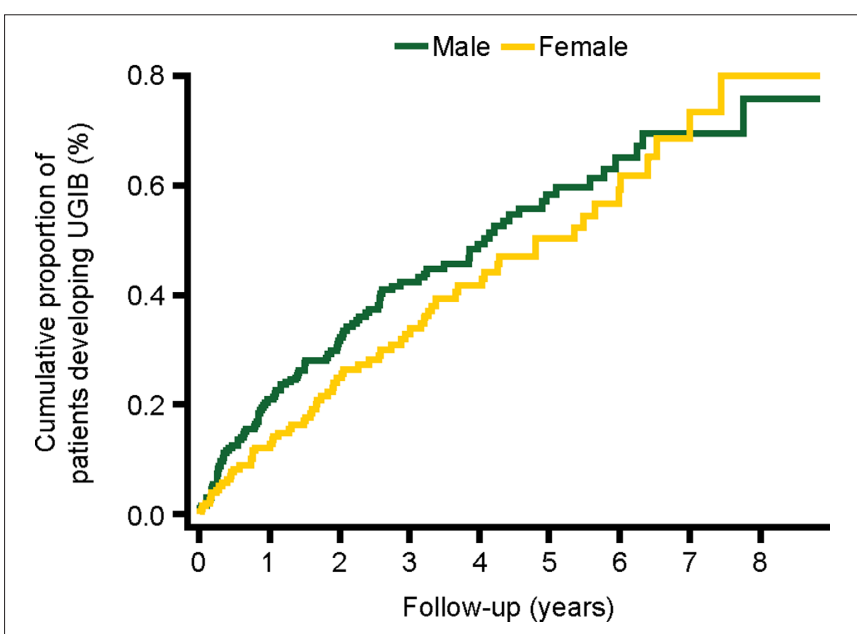

FIGURE 4 | Cumulative proportion of patients developing UGIB, stratified by sex. UGIB, upper gastrointestinal bleeding. 


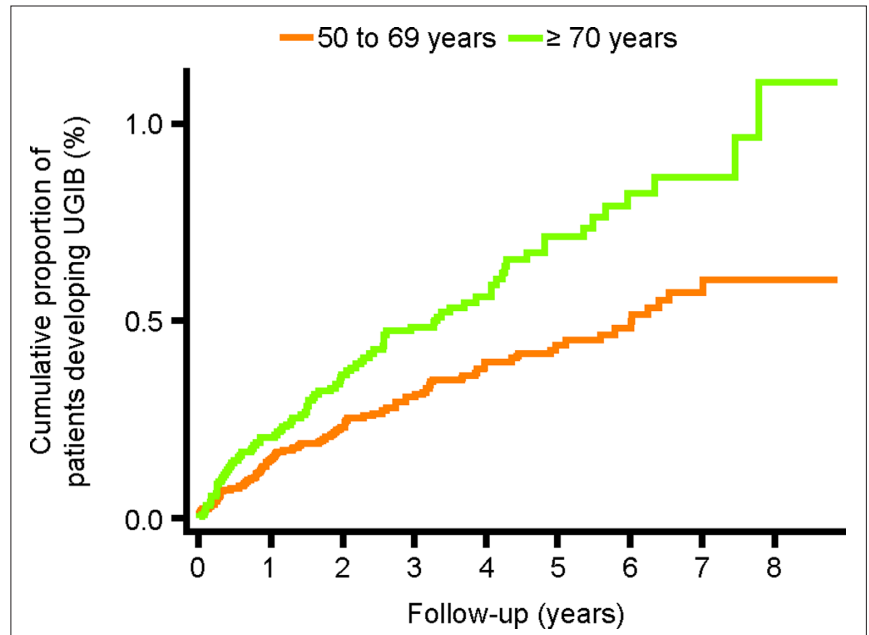

FIGURE 5 | Cumulative proportion of patients developing UGIB, stratified by age at start date. UGIB, upper gastrointestinal bleeding.

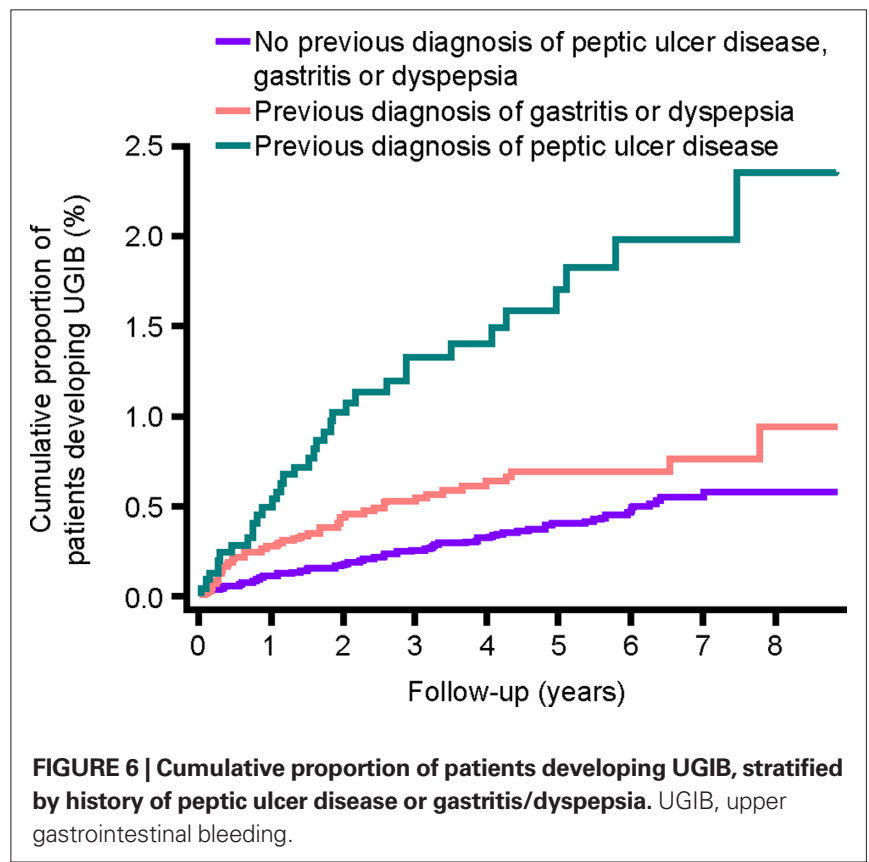

UGIB by the reason for discontinuation, individuals discontinuing ASA due to safety concerns had a RR of 2.18 (95\% CI, 0.80-5.95) compared with current users, while discontinuation for other reasons (primarily non-adherence) was associated with an RR of 0.52 (95\% CI, 0.28-0.99).

Table 4 shows the RR of UGIB associated with various antiinflammatory drugs. Compared with non-use, an increased risk of UGIB was found in current users of NSAIDs (RR, 2.66; 95\% CI, 1.66-4.26). The increase in the risk of UGIB was higher in those who had been using NSAIDs for less than 31 days (RR, 3.49; 95\% CI, 1.49-8.18) than in those who had been using longer than 30 days (RR, 2.19; 95\% CI, 1.22-3.92) compared with non-users. No relationship was found between NSAID dose and the risk of UGIB. Concomitant users of ASA and NSAIDs had a RR of 5.90
Table 1 |The prevalence of demographic and lifestyle characteristics in individuals with UGIB and controls with no UGIB, and their association with a diagnosis of UGIB, in a cohort of low-dose ASA users.

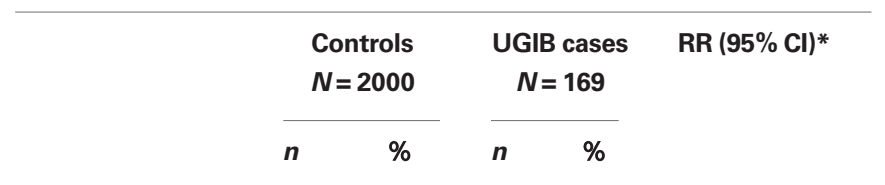

\begin{tabular}{lrrrrr}
\hline SEX & & & & & \\
Male & 1189 & 59.5 & 101 & 59.8 & NA \\
Female & 811 & 40.6 & 68 & 40.2 & NA \\
AGE (YEARS) & & & & & \\
$<65$ & 468 & 23.4 & 38 & 22.5 & NA \\
$65-74$ & 670 & 33.5 & 58 & 34.3 & NA \\
$\geq 75$ & 862 & 43.1 & 73 & 43.2 & NA
\end{tabular}

FOLLOW-UPTIME (MONTHS)

$\begin{array}{lrrrrl}\text { 0-6 } & 439 & 21.9 & 39 & 23.1 & \text { NA } \\ 7-12 & 257 & 12.8 & 23 & 13.6 & \text { NA } \\ 12-23 & 428 & 21.4 & 35 & 20.7 & \text { NA } \\ 24-47 & 484 & 24.2 & 41 & 24.3 & \text { NA } \\ \geq 48 & 392 & 19.6 & 31 & 18.3 & \text { NA } \\ \text { SMOKING STATUS } & & & & & \\ \text { Never } & 878 & 43.9 & 59 & 34.9 & 1(-) \\ \text { Current } & 276 & 13.8 & 28 & 16.6 & 1.33(0.80-2.21) \\ \text { Former } & 803 & 40.2 & 75 & 44.4 & 1.18(0.81-1.73) \\ \text { Unknown } & 43 & 2.1 & 7 & 4.1 & 2.67(1.08-6.59)\end{array}$

\begin{tabular}{lcccrl}
\multicolumn{4}{l}{ ALCOHOL USE (UNITS/WEEK) } \\
0 & 903 & 45.1 & 68 & 40.2 & $1(-)$ \\
$1-2$ & 262 & 13.1 & 26 & 15.4 & $1.49(0.91-2.44)$ \\
$3-24$ & 599 & 29.9 & 46 & 27.2 & $1.11(0.73-1.70)$ \\
$\geq 25$ & 72 & 3.6 & 12 & 7.1 & $2.96(1.43-6.15)$ \\
Unknown & 164 & 8.2 & 17 & 10.1 & $1.52(0.84-2.76)$ \\
HOSPITALIZATIONS & & & & & \\
None & 1616 & 80.8 & 112 & 66.3 & $1(-)$ \\
$1-2$ & 300 & 15.0 & 43 & 25.4 & $1.88(1.24-2.86)$ \\
$\geq 3$ & 84 & 4.2 & 14 & 8.3 & $1.56(0.79-3.05)$ \\
HISTORY OF GASTROINTESTINAL DISEASE & & \\
None & 1414 & 70.7 & 86 & 50.9 & $1(-)$ \\
Dyspepsia & 450 & 22.5 & 49 & 29.0 & $1.77(1.20-2.61)$ \\
Peptic ulcer disease & 136 & 6.8 & 34 & 20.1 & $4.59(2.87-7.33)$ \\
$\quad$ Uncomplicated & 99 & 5.0 & 22 & 13.0 & $4.18(2.44-7.15)$ \\
$\quad$ Complicated & 37 & 1.8 & 12 & 7.1 & $5.73(2.73-12.02)$ \\
\hline
\end{tabular}

${ }^{*}$ Adjusted by sex, age, follow-up time, history of peptic ulcer disease, number of $P C P$ visits, referrals and hospitalizations, and use of warfarin, ASA, clopidogrel, NSAIDs and PPIs.

Note: PCP visits, number of referrals and number of hospitalizations were collected for the year prior to the index date. A unit per alcohol: 1 unit $=10 \mathrm{ml}$ of pure ethanoll $8 \mathrm{~g}$ of alcohol). Uncomplicated ulcer diseases: peptic ulcer without complication; complicated ulcer diseases: peptic ulcer with bleeding or perforation.

ASA, acetylsalicylic acid; Cl, confidence interval; NSAID, non-steroidal anti-inflammatory drug; $P C P$, primary care practitioner; $P P I$, proton pump inhibitor; $P U$, peptic ulcer; $R R$, relative risk; UGIB, upper gastrointestinal bleeding.

(95\% CI, 2.39-14.53) compared with individuals using neither ASA nor NSAIDs. There was no significant association between oral corticosteroid use and the risk of UGIB. 
Table 2 | Medication use in individuals with UGIB and controls with no UGIB, and their association with a diagnosis of UGIB, in a cohort of low-dose ASA users.

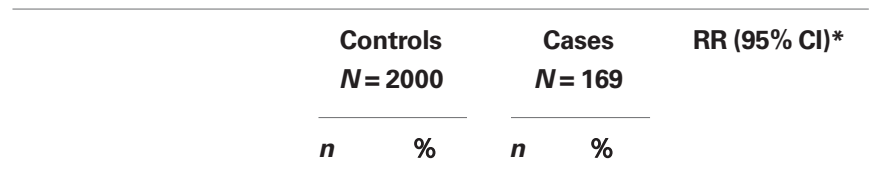

\begin{tabular}{|c|c|c|c|c|c|}
\hline \multicolumn{6}{|l|}{ LOW-DOSE ASA } \\
\hline Non-use & 186 & 9.3 & 13 & 7.7 & $1(-)$ \\
\hline Current use (0-30 days) & 1570 & 78.5 & 136 & 80.5 & $1.63(0.85-3.13)$ \\
\hline 75 mg/day & 1425 & 71.2 & 118 & 69.8 & $1.54(0.80-2.97)$ \\
\hline 150-300 mg/day & 145 & 7.2 & 18 & 10.7 & $2.65(1.17-5.97)$ \\
\hline Past use (31-365 days) & 244 & 12.2 & 20 & 11.8 & $1.31(0.59-2.88)$ \\
\hline \multicolumn{6}{|l|}{ CLOPIDOGREL } \\
\hline Non-use & 1813 & 90.6 & 139 & 82.2 & $1(-)$ \\
\hline Current use (0-30 days) & 149 & 7.4 & 22 & 13.0 & $1.90(1.12-3.22)$ \\
\hline Past use (31-365 days) & 38 & 1.9 & 8 & 4.7 & $2.40(1.04-5.55)$ \\
\hline \multicolumn{6}{|c|}{ INTERACTION WITH CLOPIDOGREL } \\
\hline $\begin{array}{l}\text { Low-dose ASA with } \\
\text { no clopidogrel }\end{array}$ & 1439 & 72.0 & 114 & 67.5 & $1(-)$ \\
\hline $\begin{array}{l}\text { Low-dose ASA } \\
\text { plus clopidogrel }\end{array}$ & 103 & 5.2 & 14 & 8.3 & $1.61(0.85-3.05)$ \\
\hline
\end{tabular}

$\begin{array}{lrrrrl}\text { WARFARIN } & 1907 & 95.3 & 161 & 95.3 & 1(-) \\ \text { Non-use } & 75 & 3.8 & 8 & 4.7 & 1.30(0.57-2.95) \\ \text { Current use (0-30 days) } & 18 & 0.9 & 0 & 0.0 & - \\ \text { Past use (31-365 days) } & & & & & \\ \text { DIPYRIDAMOLE } & 1920 & 96.0 & 161 & 95.3 & 1(-) \\ \text { Non-use } & 65 & 3.2 & 8 & 4.7 & 1.38(0.63-3.06) \\ \text { Current use (0-30 days) } & 15 & 0.8 & 0 & 0 & - \\ \text { Past use (31-365 days) } & & & & & \\ \text { NITRATES } & 1321 & 66.1 & 103 & 61.0 & 1(-) \\ \text { Non-use } & 412 & 20.6 & 36 & 21.30 & 0.93(0.61-1.42) \\ \text { Current use (0-30 days) } & 267 & 13.3 & 30 & 17.7 & 1.20(0.76-1.90) \\ \text { Past use (31-365 days) } & & & & & \end{array}$

*Adjusted by sex, age, follow-up time, history of peptic ulcer disease, number of PCP visits, referrals and hospitalizations, and use of warfarin, ASA, clopidogrel, NSAIDs, and PPIs.

Note: Data on PCP visits, number of referrals and number of hospitalizations were collected for the year prior to the index date. Data on comorbidity was collected for any time before the start date.

ASA, acetylsalicylic acid; $\mathrm{Cl}$, confidence interval; NSAID, non-steroidal anti-inflammatory drug; $P C P$, primary care practitioner; $P$ PI, proton pump inhibitor; $P U$, peptic ulcer; $R R$, relative risk; UGIB, upper gastrointestinal bleeding.

Table 5 presents the RR of UGIB associated with acidsuppressing drugs. Current PPI users who initiated this treatment at or before the start date had a significantly reduced risk of UGIB compared with non-users (RR, 0.56; 95\% CI, 0.33-0.96). Current PPI users who initiated this therapy after the start date had an increased risk of UGIB, presumably due to confounding by indication. The reduced risk of UGIB was found among current PPI users who were receiving a medium or high daily dose (RR, 0.40; 95\% CI, 0.20-0.78), but was not observed among users of a low daily dose (RR, 1.00; 95\% CI, 0.47-2.12). No significant association was found between $\mathrm{H}_{2} \mathrm{RA}$ use and the risk of UGIB (RR, 1.07; 95\% CI, 0.47-2.42).
Table 3 |The prevalence of low-dose ASA discontinuation in individuals with UGIB and controls with no UGIB, and its association with a diagnosis of UGIB, in a cohort of low-dose ASA users.

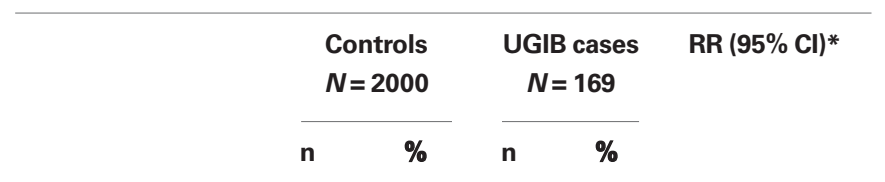

\begin{tabular}{lrrrrl}
\hline \multicolumn{2}{l}{ LOW-DOSE ASA STATUS } & & & & \\
Current users & 1494 & 74.7 & 131 & 77.5 & $1(-)$ \\
Recent discontinuers & 263 & 13.1 & 19 & 11.2 & $0.71(0.42-1.20)$ \\
Past discontinuers & 57 & 2.9 & 6 & 3.6 & $1.03(0.40-2.62)$
\end{tabular}

REASON FOR DISCONTINUATION (AMONG RECENT DISCONTINUERS)

$\begin{array}{lrrrrr}\text { Not safety related }^{\dagger} & 244 & 12.2 & 12 & 7.1 & 0.52(0.28-0.99) \\ \text { Safety related } & 19 & 0.9 & 7 & 4.1 & 2.18(0.80-5.95)\end{array}$

*Adjusted by sex, age, follow-up time, history of peptic ulcer disease, number of $P C P$ visits, referrals and hospitalizations, and use of warfarin, ASA, clopidogrel, NSAIDs and PPIs.

${ }^{+}$Includes discontinuation due to lack of efficacy, non-adherence or OTC ASA use.

Note: The remaining group not included in the table were all individuals who had non-use of ASA within the year prior to index date (186 controls and 13 cases) Data on PCP visits, number of referrals and number of hospitalizations were collected for the year prior to the index date. Data on comorbidity was collected for any time before the start date.

ASA, acetylsalicylic acid; $C l$, confidence interval; NSAID, non-steroidal anti-inflammatory drug; OTC, over-the-counter; PCP, primary care practitioner; PPI, proton pump inhibitor; $P U$, peptic ulcer; $R R$, incidence rate ratio; UGIB, upper gastrointestinal bleeding.

\section{DISCUSSION}

The incidence of UGIB in this cohort of low-dose ASA users was 1.12 per 1000 person-years. The incidence of UGIB was increased more than fivefold in those with a history of peptic ulcer disease, confirming the results of other studies that have shown this to be the strongest risk factor for UGIB (Hernández-Díaz and García Rodríguez, 2006; Lanas et al., 2006).

Current use of $75 \mathrm{mg}$ ASA was associated with a $50 \%$ increase in RR and use of 150-300 mg ASA with a greater than two-fold increase in the risk of UGIB compared with non-use, which is consistent with the results of previous clinical trials and observational studies (Weil et al., 1995; Kelly et al., 1996; de Abajo and García Rodríguez, 2001; García Rodríguez et al., 2001). Our study also shows that discontinuation of low-dose ASA therapy was associated with a $30 \%$ reduction in the risk of UGIB compared with continuation of therapy, suggesting that the gastric mucosa reverts to its pretreatment stage after treatment is withdrawn. When we stratified this analysis by the reason for low-dose ASA discontinuation, the reduced risk of UGIB was found among those who discontinued ASA for reasons unrelated to safety whereas a marked increase in the risk of UGIB was found among those who discontinued lowdose ASA for safety reasons. This strongly suggests a confounding by indication (reason for discontinuation) in this latter subgroup of discontinuers.

Adding to the literature on the beneficial effects of PPIs in this patient group (Lanas et al., 2000, 2006), we also found that concomitant PPI use reduces the risk of UGIB in low-dose ASA users by almost $50 \%$. The beneficial effect of PPIs was only found if they 
Table 4 | Anti-inflammatory drug use in individuals with UGIB and controls with no UGIB, and its association with a diagnosis of UGIB, in a cohort of low-dose ASA users.

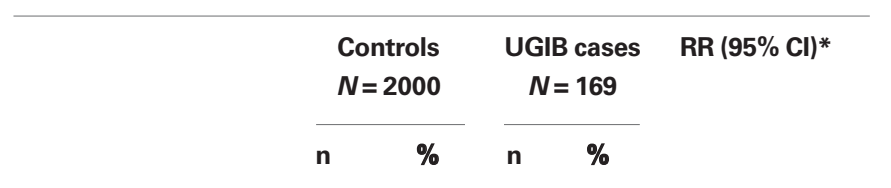

\begin{tabular}{|c|c|c|c|c|c|}
\hline \multicolumn{6}{|l|}{ NSAIDs } \\
\hline Non-use & 1607 & 80.3 & 116 & 68.6 & $1(-)$ \\
\hline Current use & 156 & 7.8 & 28 & 16.6 & $2.66(1.66-4.26)$ \\
\hline Single NSAID & 143 & 7.1 & 24 & 14.2 & $2.50(1.52-4.11)$ \\
\hline Multiple NSAIDs & 13 & 0.7 & 4 & 2.4 & $4.41(1.33-14.63$ \\
\hline Past use & 237 & 11.8 & 25 & 14.8 & $1.39(0.86-2.26)$ \\
\hline \multicolumn{6}{|c|}{ DURATION OF NSAID USE ${ }^{\dagger}$} \\
\hline$\leq 30$ days & 33 & 1.7 & 8 & 4.7 & $3.49(1.49-8.18)$ \\
\hline $31-365$ days & 64 & 3.2 & 9 & 5.3 & $2.07(0.97-4.42)$ \\
\hline$>1$ year & 46 & 2.3 & 7 & 4.1 & $2.35(1.00-5.57)$ \\
\hline \multicolumn{6}{|l|}{ NSAID DOSE ${ }^{\dagger}$} \\
\hline Low-medium & 65 & 3.2 & 13 & 7.7 & $3.13(1.61-6.06)$ \\
\hline High & 65 & 3.2 & 10 & 5.9 & $2.10(1.01-4.34)$ \\
\hline Unknown & 13 & 0.6 & 1 & 0.6 & $1.49(0.19-11.73)$ \\
\hline \multicolumn{6}{|c|}{ INTERACTION WITH NSAIDS } \\
\hline ASA without NSAID & 1260 & 63.0 & 92 & 54.4 & $1(-)$ \\
\hline ASA plus NSAID & 133 & 6.7 & 26 & 15.4 & $2.92(1.77-4.82)$ \\
\hline \multicolumn{6}{|c|}{ ORAL CORTICOSTEROIDS } \\
\hline Non-use & 1891 & 94.6 & 159 & 94.1 & $1(-)$ \\
\hline Current use (0-30 days) & 60 & 3.0 & 5 & 3.0 & $0.88(0.33-2.31)$ \\
\hline Past use (31-365 days) & 49 & 2.4 & 5 & 3.0 & $0.97(0.36-2.62)$ \\
\hline
\end{tabular}

${ }^{*}$ Adjusted by sex, age, follow-up time, history of peptic ulcer disease, number of PCP visits, referrals and hospitalizations, and use of warfarin, ASA, clopidogrel, NSAIDs, and PPIS.

${ }^{\dagger}$ Duration and dose-response was evaluated among current single NSAID users. Reference group for duration and dose-response was non-use. Specific cut-off values for dose (in mg) were as follows: aceclofenac 200, acemetacin 120, azapropazone 600, celecoxib 200, diclofenac 100, diflunisal 1500, etodolac 400 , etoricoxib 90, fenbufen 900, fenoprofen 1200, flurbiprofen 150, ibuprofen 1200, indomethacin 75, ketoprofen 150, ketorolac 30, mefenamic acid 1000, meloxicam 7.5, nabumetone 1000, naproxen 750, piroxicam 10, rofecoxib 25, sulindac 200, tenoxicam 10, tiaprofenic 600, and valdecoxib 20. Doses less than or equal to the cut-off value were grouped under low-medium doses, and doses greater than the cut-off value were grouped under high doses.

Note: Data on PCP visits, number of referrals and number of hospitalizations were collected for the year prior to the index date. Data on comorbidity was collected for any time before the start date.

ASA, acetylsalicylic acid; $\mathrm{Cl}$, confidence interval; NSAID, non-steroidal anti-inflammatory drug; OTC, over-the-counter; $P C P$, primary care practitioner; PPI, proton pump inhibitor; PU, peptic ulcer; RR, relative risk; UGIB, upper gastrointestinal bleeding.

were initiated at the same time as the low-dose ASA or before the first low-dose ASA prescription. Again this suggests a confounding by indication for PPI use in those who were not prescribed a PPI until sometime after their low-dose ASA prescription. Our data on use of H2RAs was limited and we could not assess with precision the effect of this drug class. Some studies have shown a protective effect of H2RAs on the risk of UGIB among ASA users (Lanas et al., 2007).

We also found that prescribing ASA together with other drugs, such as NSAIDs or clopidogrel, confers an increased risk of UGIB. Users of clopidogrel had an almost twofold increase in the risk
Table 5 | PPI and $\mathrm{H}_{2} R A$ use in individuals with UGIB and controls with no UGIB, and its association with a diagnosis of UGIB, in a cohort of low-dose ASA users.

\begin{tabular}{|c|c|c|c|c|c|}
\hline & \multicolumn{2}{|c|}{$\begin{array}{l}\text { Controls } \\
N=2000\end{array}$} & \multicolumn{2}{|c|}{$\begin{array}{c}\text { Cases } \\
N=169\end{array}$} & \multirow[t]{2}{*}{$\mathbf{R R}(95 \% \mathrm{Cl}) *$} \\
\hline & $\mathbf{n}$ & $\%$ & $\mathbf{n}$ & $\%$ & \\
\hline \multicolumn{6}{|l|}{ PPI USE AT INDEX DATE } \\
\hline Non-use & 1490 & 74.5 & 107 & 63.3 & $1(-)$ \\
\hline Current use (0-30 days) & 400 & 20.0 & 46 & 27.2 & $0.97(0.65-1.44)$ \\
\hline Past use (31-365 days) & 110 & 5.5 & 16 & 9.5 & $1.30(0.70-2.41)$ \\
\hline \multicolumn{6}{|l|}{ PPI USE AT START DATE } \\
\hline Non-use & 1490 & 74.5 & 107 & 63.3 & $1(-)$ \\
\hline $\begin{array}{l}\text { Current users initiating PPI } \\
\text { therapy after start date }\end{array}$ & 135 & 6.8 & 25 & 14.8 & $1.88(1.14-3.13)$ \\
\hline Current PPI users initiating & 265 & 13.2 & 21 & 12.4 & $0.56(0.33-0.96)$ \\
\hline \multicolumn{6}{|l|}{ PPI at start date } \\
\hline Low PPI dose ${ }^{\dagger}$ & 84 & 4.2 & 9 & 5.3 & $1.00(0.47-2.12)$ \\
\hline Medium/high PPI dose ${ }^{\dagger}$ & 181 & 9.0 & 12 & 7.1 & $0.40(0.20-0.78)$ \\
\hline \multicolumn{6}{|l|}{$\mathrm{H}_{2} \mathrm{RA}$ AT INDEX DATE } \\
\hline Non-use & 1885 & 94.2 & 152 & 89.9 & $1(-)$ \\
\hline Current use (0-30 days) & 84 & 4.2 & 12 & 7.1 & $1.08(0.55-2.11)$ \\
\hline Past use (31-365 days) & 31 & 1.6 & 5 & 3.0 & $1.45(0.51-4.12)$ \\
\hline \multicolumn{6}{|l|}{$\mathrm{H}_{2}$ RA AT START DATE } \\
\hline Non-use & 1885 & 94.2 & 152 & 89.9 & $1(-)$ \\
\hline $\begin{array}{l}\text { Current users initiating } \mathrm{H}_{2} \mathrm{RA} \\
\text { therapy after start date }\end{array}$ & 28 & 1.4 & 4 & 2.4 & $1.09(0.36-3.31)$ \\
\hline $\begin{array}{l}\text { Current PPI users initiating } \\
\mathrm{H}_{2} \mathrm{RA} \text { therapy at start date }\end{array}$ & 56 & 2.8 & 8 & 4.7 & $1.07(0.47-2.41)$ \\
\hline
\end{tabular}

${ }^{*}$ Adjusted by sex, age, follow-up time, history of peptic ulcer disease, number of PCP visits, referrals and hospitalizations, and use of warfarin, ASA, clopidogrel, NSAIDs and PPIs.

${ }^{+}$Specific cut-off values for daily dose (in $\mathrm{mg}$ ) were as follows: esomeprazole 10, lansoprazole 15, omeprazole 10, rabeprazole 10, pantoprazole $10 \mathrm{mg}$. Doses less than or equal to the cut-off value were grouped under low doses, and doses greater than the cut-off value were grouped under medium/high doses.

ASA, acetylsalicylic acid; $\mathrm{Cl}$, confidence interval; $\mathrm{H}_{2} R A$, histamine-2 receptor antagonist; NSAID, non-steroidal anti-inflammatory drug; $P C P$, primary care practitioner; $P P I$, proton pump inhibitor; $P U$, peptic ulcer; $R R$, relative risk; UGIB, upper gastrointestinal bleeding.

of UGIB compared with non-users, which supports the results of other case-control studies (Hallas et al., 2006; Ibáñez et al., 2006). The inhibition of platelet aggregation by clopidogrel, in addition to causing a vascular homeostatic imbalance, could have a role in ulcer healing (Ma et al., 2001; Lanas and Scheiman, 2007), leading to a relapse of latent ulcers.

NSAID use was also associated with an increase in the risk of UGIB in users of low-dose ASA, but this increase was smaller than that found in studies of the general population (Hernández-Díaz and García Rodríguez, 2002). This was probably due to our cohort being restricted to individuals taking low-dose ASA for secondary prevention of cardiovascular events. These individuals already have complete inhibition of thromboxane-mediated platelet aggregation afforded by aspirin, which means that the increase in the risk of UGIB in these individuals due to concomitant use of low-dose ASA and NSAIDs is most likely a result of directly induced gastrointestinal ulceration. 
Our study has several strengths and limitations. A key strength is that the records of potential cases (including free-text comments) were manually reviewed. In a previous study, this method of case ascertainment was shown to have a positive predictive value of almost 95\% when PCPs were contacted in order to confirm the diagnosis (García Rodríguez and Barreales Tolosa, 2007). In addition, although our study is retrospective, data were recorded prospectively by the PCP before the episode of interest. We tried to minimize any residual confounding; however, some level of misclassification is unavoidable in a database of this size. However, non-differential misclassification should have biased our estimates of effect toward the null and does not explain the strong associations and dose-response relationships observed in our study. Also, the lack of systematic recording of over-the-counter ASA is another source of misclassification, however it should be noted that individuals aged over 60 years (the majority of our study members) are eligible for free prescriptions, which indicates a high likelihood to collect the prescription. Despite the large size of the total study cohort, the number of UGIB cases was small, which may have affected the results of some of the sub-analyses as the study of dose and duration-response according to ASA use. Another limitation is the lack of consistent recording of $H$. pylori infection in THIN, which made it impossible to isolate the effect of $H$. pylori and $H$. pylori eradication on the risk of UGIB.

\section{REFERENCES}

Antithrombotic Trialists' Collaboration. (2002). Collaborative meta-analysis of randomised trials of antiplatelet therapy for prevention of death, myocardial infarction, and stroke in high risk patients. BMJ 324, 71-86.

Bhatt, D. L., Scheiman, J., Abraham, N. S., Antman, E. M., Chan, F. K., Furberg, C. D., Johnson, D. A., Mahaffey, K. W., and Quigley, E. M. (2008). ACCF/ACG/AHA 2008 expert consensus document on reducing the gastrointestinal risks of antiplatelet therapy and NSAID use: a report of the American College of Cardiology Foundation Task Force on Clinical Expert Consensus Documents. Circulation 118, 1894-1909.

Bourke, A., Dattani, H., and Robinson, M. (2004). Feasibility study and methodology to create a quality-evaluated database of primary care data. Inform. Prim. Care 12, 171-177.

Collet, J. P., Montalescot, G., Blanchet, B., Tanguy, M.L., Golmard, J.L., Choussat, R., Beygui, F., Payot, L., Vignolles, N., Metzger, J. P., and Thomas, D. (2004). Impact of prior use or recent withdrawal of oral antiplatelet agents on acute coronary syndromes. Circulation 110, 2361-2367.

de Abajo, F. J., and García Rodríguez, L. A. (2001). Risk of upper gastrointestinal bleeding and perforation associated with low-dose aspirin as plain and enteric-coated formulations. BMC Clin. Pharmacol. 1, 1.
First Data Bank. (2010). MULTILEX for Primary Care. Available from: http:// www.firstdatabank.co.uk/uploads/ files/MultilexDDF\%20for \%20 Primary\%20Care.pdf (Accessed 25 January 2010).

García Rodríguez, L. A., and Barreales Tolosa, L. (2007). Risk of upper gastrointestinal complications among users of traditional NSAIDs and COXIBs in the general population. Gastroenterology 132, 498-506.

García Rodríguez, L. A., Cea Soriano, L., Martín-Merino, E., and Johansson, S. (2009). Discontinuation of lowdose acetylsalicylic acid treatment for secondary prevention of cardiovascular outcomes is associated with an increased risk of myocardial infarction. Circulation 120, S405.

García Rodríguez, L. A., HernándezDíaz, S., and de Abajo, F. J. (2001). Association between aspirin and upper gastrointestinal complications: systematic review of epidemiologic studies. Br. J. Clin. Pharmacol. 52, 563-571.

Hallas, J., Dall, M., Andries, A., Andersen, B. S., Aalykke, C., Hansen, J. M., Andersen, M., and Lassen, A. T. (2006). Use of single and combined antithrombotic therapy and risk of serious upper gastrointestinal bleeding: population based case-control study. BMJ 333, 726. L.A. (2002). Incidence of serious upper gastrointestinal bleeding/perforation
Hernández-Díaz, S., and García Rodríguez,

In conclusion, the results of the present study provide additional evidence that a history of peptic ulcer disease increases the risk of UGIB among new users of low-dose ASA for secondary prevention of cardiovascular and cerebrovascular events. In addition, these data support the finding that combining ASA with NSAIDs or clopidogrel increases further the risk of UGIB; while prescribing a PPI when initiating low-dose ASA therapy reduces the risk of UGIB.

Discontinuation of low-dose ASA also reduces the risk of UGIB. However, individuals with a history of cardiovascular events who discontinue treatment with low-dose ASA are known to be at increased risk of myocardial infarction (García Rodríguez et al., 2009), transient ischemic attack (Maulaz et al., 2005) and death (Collet et al., 2004) compared with those who continue treatment. When prescribing low-dose ASA to individuals at high gastrointestinal risk, clinicians should therefore weigh the potential benefit of co-prescribing a PPI to reduce the burden of gastrointestinal disease in these patients.

\section{ACKNOWLEDGMENTS}

This study was funded by an unrestricted research grant from AstraZeneca R\&D Mölndal. We thank Dr Catherine Hill of Oxford PharmaGenesis ${ }^{\mathrm{TM}}$ Ltd, who provided editing support funded by AstraZeneca R\&D Mölndal.

in the general population: review of epidemiologic studies. J. Clin. Epidemiol. 55, 157-163.

Hernández-Díaz, S., and García Rodríguez, L. A. (2006). Cardioprotective aspirin users and their excess risk of upper gastrointestinal complications. BMC Med. 4, 22.

Ibáñez, L., Vidal, X., Vendrell, L., Moretti, U., and Laporte, J. R. (2006). Upper gastrointestinal bleeding associated with antiplatelet drugs. Aliment. Pharmacol. Ther. 23, 235-242.

Kelly, J. P., Kaufman, D. W., Jurgelon, J. M., Sheehan, J., Koff, R. S., and Shapiro, S. (1996). Risk of aspirin-associated major upper-gastrointestinalbleeding with enteric-coated or buffered product. Lancet 348, 1413-1416.

King, S. B., 3rd, Smith, S. C., Jr., Hirshfeld, J. W., Jr., Jacobs, A. K., Morrison, D. A., Williams, D. O., Feldman, T. E., Kern, M. J., O’Neill, W. W., Schaff, H. V., Whitlow, P. L., Adams, C. D., Anderson, J. L., Buller, C. E., Creager, M. A., Ettinger, S. M., Halperin, J. L., Hunt, S.A., Krumholz, H. M., Kushner, F. G., Lytle, B. W., Nishimura, R., Page, R. L., Riegel, B., Tarkington, L. G., and Yancy, C. W. (2008). 2007 Focused update of the ACC/AHA/SCAI 2005 guideline update for percutaneous coronary intervention: a report of the American college of cardiology/ American heart association task force on practice guidelines: 2007 writing group to review new evidence and update the ACC/AHA/SCAI 2005 guideline update for percutaneous coronary intervention, writing on behalf of the 2005 writing committee. Circulation 117, 261-295.

Lanas, A., Bajador, E., Serrano, P., Fuentes, J., Carreno, S., Guardia, J., Sanz, M., Montoro, M., and Sainz, R. (2000). Nitrovasodilators, low-dose aspirin, other nonsteroidal antiinflammatory drugs, and the risk of upper gastrointestinal bleeding. N. Engl. J. Med. 343, 834-839.

Lanas, A., García Rodríguez, L. A., Arroyo, M. T., Bujanda, L., Gomollón, F., Forné, M., Aleman, S., Nicolas, D., Feu, F., González-Pérez, A., Borda, A., Castro, M., Poveda, M. J., and Arenas, J. (2007). Effect of antisecretory drugs and nitrates on the risk of ulcer bleeding associated with nonsteroidal anti-inflammatory drugs, antiplatelet agents, and anticoagulants. Am. J. Gastroenterol. 102, 507-515.

Lanas, A., García Rodríguez, L. A., Arroyo, M. T., Gomollón, F., Feu, F., GonzálezPérez, A., Zapata, E., Bástida, G., Rodrigo, L., Santolaria, S., Güell, M., de Argila, C. M., Quintero, E., Borda, F., and Piqué, J. M. (2006). Risk of upper gastrointestinal ulcer bleeding associated with selective cyclo-oxygenase-2 inhibitors, traditional non-aspirin non-steroidal anti-inflammatory drugs, aspirin and combinations. Gut 55, 1731-1738.

Lanas, A., and Scheiman, J. (2007). Lowdose aspirin and upper gastrointestinal damage: epidemiology, prevention 
and treatment. Curr. Med. Res. Opin. 23, 163-173.

Lewis, J. D., Schinnar, R., Bilker, W. B., Wang, X., and Strom, B. L. (2007). Validation studies of the health improvement network (THIN) database for pharmacoepidemiology research. Pharmacoepidemiol. Drug. Saf. 16, 393-401.

Ma, L., Elliott, S. N., Cirino, G., Buret, A., Ignarro, L. J., and Wallace, J. L. (2001). Platelets modulate gastric ulcer healing: role of endostatin and vascular endothelial growth factor release. Proc. Natl. Acad. Sci. U.S.A. 98, 6470-6475.

Maulaz, A. B., Bezerra, D. C., Michel, P., and Bogousslavsky, J. (2005). Effect of discontinuing aspirin therapy on the risk of brain ischemic stroke. Arch. Neurol. 62, 1217-1220.

O’Neil, M., Payne, C., and Read, J. (1995). Read Codes Version 3: a user led terminology. Methods Inf. Med. 34, 187-192.
Patrono, C., García Rodríguez, L. A., Landolfi, R., and Baigent, C. (2005). Low-dose aspirin for the prevention of atherothrombosis. N. Engl. J. Med. 353, 2373-2383.

Sacco, R. L., Adams, R., Albers, G., Alberts, M. J., Benavente, O., Furie, K., Goldstein, L. B., Gorelick, P., Halperin, J., Harbaugh, R., Johnston, S. C., Katzan, I., Kelly-Hayes, M., Kenton, E. J., Marks, M., Schwamm, L. H., and Tomsick, T. (2006). Guidelines for prevention of stroke in patients with ischemic stroke or transient ischemic attack: a statement for healthcare professionals from the American Heart Association/American Stroke Association Council on Stroke: co-sponsored by the Council on Cardiovascular Radiology and Intervention: the American Academy of Neurology affirms the value of this guideline. Stroke 37, 577-617.

Smith, S.C. Jr., Allen, J., Blair, S.N., Bonow, R. O., Brass, L. M., Fonarow, G. C.,
Grundy, S. M., Hiratzka, L., Jones, D., Krumholz, H. M., Mosca, L., Pearson, T., Pfeffer, M. A., and Taubert, K. A. (2006). AHA/ACC guidelines for secondary prevention for patients with coronary and other atherosclerotic vascular disease: 2006 update endorsed by the National Heart, Lung, and Blood Institute. J. Am. Coll. Cardiol. 47, 2130-2139.

Stuart-Buttle, C.D., Read,J.D., Sanderson, H. F., and Sutton, Y. M. (1996). A language of health in action: read Codes, classifications and groupings. Proc. AMIA. Annu. Fall Symp. 75-79.

Weil, J., Colin-Jones, D., Langman, M., Lawson, D., Logan, R., Murphy, M., Rawlins, M., Vessey, M., and Wainwright, P. (1995). Prophylactic aspirin and risk of peptic ulcer bleeding. BMJ 310, 827-830.

Conflict of Interest Statement: Dr García Rodríguez and Cea Soriano work for CEIFE, which has received research funding from AstraZeneca R\&D Mölndal.

Received: 25 June 2010; paper pending published: 29 June 2010; accepted: 13 September 2010; published online: 14 October 2010.

This article was submitted to Frontiers in Inflammation Pharmacology, a specialty of Frontiers in Pharmacology.

Citation: Cea Soriano L and García Rodríguez LA (2010) Risk of upper gastrointestinal bleeding in a cohort of new users of low-dose ASA for secondary prevention of cardiovascular outcomes. Front. Pharmacol. 1:126. doi: 10.3389/ fphar.2010.00126

Copyright $\odot 2010$ Cea Soriano and García Rodríguez. This is an open-access article subject to an exclusive license agreement between the authors and the Frontiers Research Foundation, which permits unrestricted use, distribution, and reproduction in any medium, provided the original authors and source are credited. 